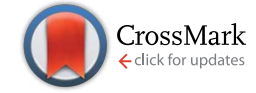

Cite this: J. Mater. Chem. A, 2016, 4, 14729

Received 28th July 2016 Accepted 17th August 2016

DOI: 10.1039/c6ta06446a

www.rsc.org/MaterialsA

\section{Recruiting physisorbed water in surface polymerization for bio-inspired materials of tunable hydrophobicity $\dagger$}

\author{
S. Oyola-Reynoso, ${ }^{a}$ I. D. Tevis, ${ }^{a}$ J. Chen, ${ }^{\text {ade }}$ B. S. Chang, ${ }^{\text {ae }}$ S. Çinar, ${ }^{a}$ J.-F. Bloch ${ }^{\text {b }}$ \\ and M. M. Thuo*acde
}

Chemical grafting has been widely used to modify the surface properties of materials, especially surface energy for controlled wetting, because of the resilience of such coatings/modifications. Reagents with multiple reactive sites have been used with the expectation that a monolayer will form. The step-growth polymerization mechanism, however, suggests the possibility of gel formation for hydrolyzable moieties in the presence of physisorbed water. In this report, we demonstrated that using alkyltrichlorosilanes (trivalent [i.e., 3 reactive sites]) in the surface modification of a cellulosic material (paper) does not yield a monolayer but rather gives surface-bound particles. We infer that the presence of physisorbed (surface-bound) water allows for polymerization (or oligomerization) of the silane prior to its attachment on the surface. Surface energy mismatch between the hydrophobic tails of the growing polymer and any unreacted bound water leads to the assembly of the polymerizing material into spherical particles to minimize surface tension. By varying paper grammage $\left(16.2-201.4 \mathrm{~g} \mathrm{~m}^{-2}\right)$, we varied the accessible surface area and thus the amount of surface-adsorbed water, allowing us to control the ratio of the silane to the bound water. Using this approach, polymeric particles were formed on the surface of cellulose fibers ranging from $\sim 70 \mathrm{~nm}$ to a film. The hydrophobicity of the surface, as determined by water contact angles, correlates with particle sizes $(p<0.001$, Student's $t$-test), and, hence, the hydrophobicity can be tuned (contact angle between $94^{\circ}$ and $149^{\circ}$ ). Using a model structure of a house, we demonstrated that as a result of this modification, paper-based houses can be rendered self-cleaning or tolerant to surface running water. In another application, we demonstrated that the felicitous choice of architectural design allows for the hydrophobic paper to be used for water harvesting.

\section{Introduction}

The Namib Desert beetle, Stenocara gracilipes, survives in xeric environments, in part due to its ability to harvest water from fog. ${ }^{1-3}$ The surface structure of the wing cases of these beetles is biphobic - that is, it has hydrophilic bumps that aid in water droplet adhesion/collection and hydrophobic valleys that act as collection channels for the water droplets to roll towards the beetle's mouth., ${ }^{\mathbf{1 , 5} 5}$ These beetles have inspired the development

${ }^{a}$ Department of Material Science and Engineering, Iowa State University Ames, IA 50011, USA. E-mail: mthuo@iastate.edu

${ }^{b}$ University of Grenoble, 461 Rue de la Papeterie, 38402 Saint-Martin-d'Heres, France ${ }^{c}$ Biopolymer and Biocomposites Research Team, Center for Bioplastics and Biocomposites, Iowa State University, Ames, Iowa 50011, USA

${ }^{d}$ Micro-electronic Research Center, Iowa State University, 133 Applied Sciences Complex I, 1925 Scholl Road, Ames, Iowa 50011, USA

${ }^{e}$ Division of Materials Science and Engineering, Ames Laboratory, Ames, Iowa 50011, USA

$\dagger$ Electronic supplementary information (ESI) available: Non-colored focused ion beam images for surface particles, X-ray diffraction, and infra-red and SEM images of high density paper. See DOI: 10.1039/c6ta06446a of various materials. ${ }^{3,6,7}$ Most of these materials often require sophisticated nano-patterning, expensive equipment, and skilled labor forces to fabricate. ${ }^{3,6,8-10}$ Biphobic surfaces are important in controlled liquid condensation and delivery beyond survival in xeric environments. The ability to fabricate materials with these complex structures has been studied for anticorrosion, ${ }^{\mathbf{8 1 1}}$ anti-biofouling, electrowetting, and drag reduction as well as fog collection. ${ }^{12}$ Many of these technologies rely on physisorbed coatings to impart hydrophobicity and do not benefit from the often more robust chemical grafting to increase the material's longevity during operation. ${ }^{3, \mathbf{6 , 8 , 9 , 1 2 - 1 6}}$ Resilience of superhydrophobic coatings is crucial for their use in preventing water contamination, surface erosion, and friction and for imparting corrosion protection; hence the need for covalent chemical attachment of the coating. ${ }^{17,18}$ In the case of covalently coupled modifiers, the use of readily hydrolyzable groups, such as esters and carbamates, renders the coating(s) labile, and better approaches are therefore needed. ${ }^{19}$

A well-understood chemical transformation coupled with concomitant self-assembly is potentially a feasible approach to alter both the surface chemistry and texture of a material 
surface for tunable hydrophobicity. Alkyltrihalosilanes ( $\left.\mathrm{RSiX}_{3}\right)$ have been widely used in creating hydrophobic surfaces but there is no consensus as to the resulting product(s). ${ }^{16,20-28}$ The reaction of trihalosilanes with material surfaces has been hypothesized to lead to (i) well-ordered single-molecule monolayers, (ii) a cross-linked monolayer either fully or partially attached to the surface, and (iii) the formation of short-chain silsesquioxanes, cyclic oligomers of about 2-5 nm. ${ }^{16,26,29-36}$ We believe that the lack of consensus may, in part, be due to challenges in controlling surface-bound water.

We hypothesized that the controlled reaction of a surfactant molecule, such as an alkyl trichlorosilane, through chemical vapor deposition with a material bearing a hydrophilic surface would lead to the formation of particles of controlled size but stochastically distributed across the surface. The presence of adsorbed water on a hydrophilic surface implies that the alkyl trichlorosilane oligomerizes before it comes into contact with the material's surface. The oligomerization recruits surface-adsorbed water as a comonomer in a step-growth polymerization process (further discussion is given in the ESI $\dagger$ ). To test this hypothesis, we exposed ultraflat $\mathrm{Au}$ (root mean square roughness $=0.45 \mathrm{~nm}$ ) and an atomically flat silicon wafer, with its native oxide, to perfluorooctyl trichlorosilane vapor. We observed that, as predicted, the surface morphology and wettability were altered, but, more importantly, spherical particles of different sizes were formed (Fig. 1A-D).
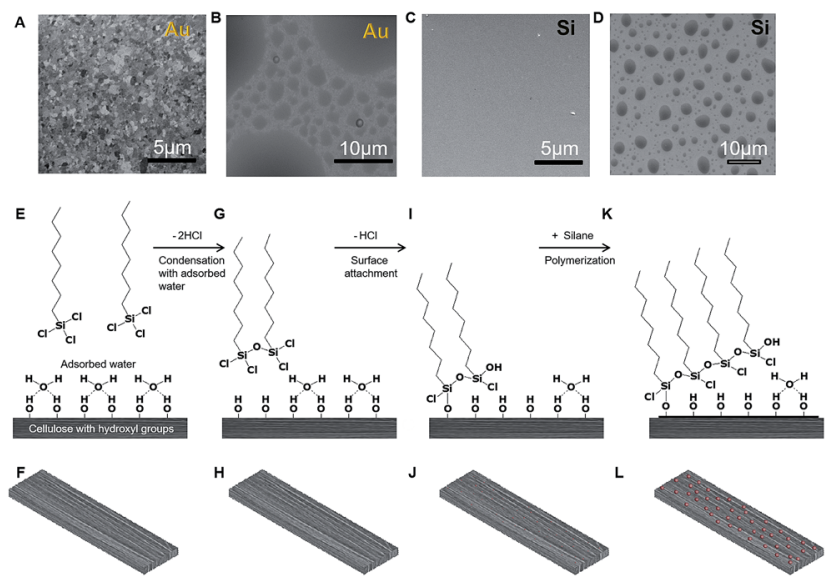

Fig. 1 Surface modification with alkyltrichlorosilane vapors in vacuo: ultraflat $A u(A)$ before and (B) after exposure to silane, and atomically flat Si wafer (C) before and (D) after exposure to silane. Exposure leads to significant changes in surface morphology. Schematic illustration of the hypothetical stepwise process of hydroxyl groups reacting with alkyltrichlorosilane vapor to form hydrophobic particles on the surface: ( $E$ and F) alkyltrichlorosilane molecules are vaporized and come into contact with the surface of the paper with its adsorbed water. ( $G$ and $H$ ) The silane molecules then react with surfaceadsorbed water to form an oligomer. (I and J) The oligomerized alkylsilane molecule reacts with surface hydroxyl groups of the cellulose to attach to the paper. ( $K$ and $L$ ) The chemisorbed polymer particles grow further, leading to an increase in size as any remaining surface water is chemically removed by the silane. More water can diffuse from the core of the paper fibers to the surface, allowing the particles to continue growing.
We envision this surface modification to occur in a fourstage surface polymerization covalent grafting process: (i) oligomerization of the alkyl silane vapor through reaction with ambient water vapor (where applicable) and eventually surface-bound water to create a macromonomer (Fig. 1G and $\mathrm{H}$ ); (ii) self-assembly of the oligomeric silanes due to the presence of adsorbed water (i.e., before all surface-bound water has been chemically removed, a large surface energy mismatch between the hydrophobic tails and the remaining bound water induces self-assembly); (iii) attachment of the oligomeric alkyl silane (macromonomers) on the surface (Fig. 1I and J), which is likely to occur from the edge of the growing oligomer particle analogous to structures inferred by Spencer and co-workers using spectroscopy; ${ }^{34,37}$ and (iv) an increase in the degree of polymerization with further crosslinking and particle growth to produce polymer particles. Continued growth will lead to confluence over the total surface (Fig. 1K and L). For clarity and brevity, we illustrate the process using a single layer of molecules; however, with the formation of a self-assembled polymeric particle, it is unlikely that the oligomerized silane is always attached on the surface as a monolayer. A three-dimensional assembly that grows beyond the range of silsesquioxanes is expected, but the presence of bound water limits the extent of this growth. To test our hypothesis, we adopted paper as a platform material and alkyltrichlorosilanes as the monomers/reagents. Paper is ubiquitous and has gained interest as a structural material (housing) in bioanalytical devices, packaging, printing, and many other areas that could benefit from controlled surface energy. ${ }^{38-44}$ On paper, surface-bound water can be tuned by altering its available surface area through grammage (fiber density).

\section{Experimental}

\section{Materials}

Trichloro( $1 H, 1 H, 2 H, 2 H$-perfluorooctyl)silane (97\%) and octyltrichlorosilane (97\%) were purchased from Sigma-Aldrich and used as received. Chromatography paper \#1 (Whatman), filter paper \#1 (Whatman), NYX blotting paper (NYX Cosmetics), and cardstock paper (Georgia Pacific) were used as substrates.

\section{Surface modification}

To fabricate the hydrophobic paper, pre-cut papers were placed in a clean, dry desiccator (kept dry using Drierite, with an indicator placed on the bottom) followed by $\sim 0.1 \mathrm{~mL}$ of requisite alkylsilane in a $10 \mathrm{~mL}$ dram vial. The desiccator was evacuated ( $\sim 30 \mathrm{mmHg}$ pressure), sealed, and placed in an oven that had been preheated to $95{ }^{\circ} \mathrm{C}$. After a predetermined time, the desiccator was removed from the oven and the paper samples were also removed to be tested for hydrophobicity, while equivalent samples were used for characterization. The reaction was performed with octyltrichlorosilane (97\% Sigma-Aldrich) or its fluorinated analog, trichloro $(1 H, 1 H, 2 H, 2 H$-perfluorooctyl) silane (97\% Sigma-Aldrich). 


\section{Contact angle measurement}

Contact angle measurements were performed with a Ramé-Hart 200 (p/n 200-U1) goniometer using $1 \mu \mathrm{L}$ of deionized water. Samples were stabilized and flattened on a glass slide. Contact angle images were collected with a high-resolution camera and analyzed with DROPimage software.

\section{Scanning electron microscopy (SEM)}

A Zeiss Supra55VP field emission SEM was used to examine the micro- and nanostructures of the paper substrates and to determine the elemental composition (using Energy Dispersive X-ray Spectroscopy [EDS]) of their surfaces. Samples were imaged using an electron beam with an accelerating voltage of $1 \mathrm{kV}$ and a working distance of $5.4 \mathrm{~mm}$. Images were collected using an Everhart-Thornley secondary electron detector. An FEI Quanta 250 FE-SEM microscope was also used for surface analysis where samples were imaged using an accelerating voltage of $1 \mathrm{kV}$ and a working distance of $11 \mathrm{~mm}$. The elemental composition at various accelerating voltages was determined using energy-dispersive X-ray spectroscopy with a silicon drift detector at an accelerating voltage of $10 \mathrm{kV}$, a working distance of $8.6 \mathrm{~mm}, 1 \mathrm{~nm}$ sputtered gold coating, $128 \times 100$ resolution, 256 frames, a $100 \mathrm{~ms}$ dwell time, and corrected for drift.

\section{Scanning electron microscopy-focused ion beam (SEM-FIB)}

A Zeiss NVision 40 dual-beam SEM-FIB was used to image the formed particles on paper and mill them to inspect their interiors. Imaging was performed at $5 \mathrm{kV}$ with a working distance of $4.7 \mathrm{~mm}$ tilted at a $54^{\circ}$ angle. Images were collected using the Everhart-Thornley secondary electron detector. A focused ion beam of gallium ions was used to mill away a rectangular area directly over half of the formed fluoroalkylsilane particles, using an accelerating voltage of $30 \mathrm{kV}$ and an ion current of $10 \mathrm{pA}$.

\section{X-ray diffraction (XRD)}

XRD was performed with a Rigaku Ultima IV powder diffractometer to examine changes in the crystallinity of the material at a scanning rate of $1^{\circ}$ per minute. Most polymeric materials, such as cellulose, are semi-crystalline and as such, X-ray diffraction peaks are expected..$^{\mathbf{4 5 , 4 6}}$ The diffractograms of our dense NYX blotting paper indicated some degree of crystallinity, with an intense peak corresponding to one commonly attributed to cellulose I (see ESI S3†).

\section{X-ray photo-electron spectroscopy (XPS)}

XPS was performed on untreated and treated paper samples using a Thermo Scientific K-Alpha XPS with an AlK $\alpha$ X-ray source. A $100 \mu \mathrm{m}$ spot size was used for all analysis. A survey scan was performed to qualitatively identify elements on the surface of the paper using a pass energy of $200 \mathrm{eV}$, an average of 5 scans, a dwell time at each step of $10 \mathrm{~ms}$, and an energy step size of $1.0 \mathrm{eV}$, with an electron flood gun active to prevent charging. This fast scan should lessen any damage caused by X-ray exposure. After the identification of elements present in the sample, quantification of the elements was performed at each element's signature peak using a pass energy of $50 \mathrm{eV}$, an average of 8 scans, a dwell time at each step of $50 \mathrm{~ms}$, and an energy step size of $0.1 \mathrm{eV}$, with an electron flood gun active to prevent charging. Quantification of all elements and the background correction of the Si2p peak were performed using the Advantage software. Peaks were identified manually and fit using the Advantage program. XPS spectra were corrected to $284.8 \mathrm{eV}$ for adventitious C1s. Silicon $2 \mathrm{p}$ peak regions were smoothed using a $1 \mathrm{eV}$ moving average.

\section{Fourier transform infrared spectroscopy (FTIR)}

FTIR was performed on a Thermo Nicolet 380 FTIR system with the Smart Orbit accessory with a diamond ATR crystal and DTGS detector. Peak finding and subtraction were performed using the OMNIC software.

\section{Results and discussion}

To test our hypothesis, papers of different grammage and with or without fillers were used. The selected papers were all hydrophilic (completely wet and wick water) prior to our treatment. The silane reagents were maintained under dry conditions, and only a small amount $(\sim 0.5 \mathrm{~mL})$ was added to the reaction chamber immediately before initiating the reaction. All reactions were run at reduced pressure and elevated temperatures $\left(70-90{ }^{\circ} \mathrm{C}\right)$ to mitigate the effect of ambient humidity and to control the amount of surface-bound water on the cellulose.

\section{Adsorbed surface water as a cross-linker in surface step- growth polymerization of octyltrichlorosilane}

Three types of papers were used to capture the effect of surfacebound water, polycarbonate fillers, and grammage on the treatment of paper with alkyl trichlorosilanes. Fig. 2 shows SEM images of the native paper (left column) and the treated analogs (middle and right columns). As expected, different types of papers gave varied results upon treatment with the alkylsilanes (ESI is shown in Fig. S1†). When a primarily cellulosic material (Whatman chromatography paper \#1) was subjected to silanization, there were no observable differences in the fiber surface texture between the control (Fig. 2A(i)) and the treated paper (Fig. 2A(ii)), even though the paper became hydrophobic. The lack of differences in the surface texture between the treated and untreated paper can be due to the formation of either a monolayer ${ }^{47-50}$ or a cross-linked film..$^{34,37,49}$

\section{Effect of filler materials}

Cardstock paper, which contains precipitated calcium carbonate (PCC) fillers, ${ }^{51,52}$ was used to test the effect of additives on the reaction. Fig. $2 \mathrm{~B}(\mathrm{i})$ shows the native cardstock paper with a few clusters of the granular PCC filler. Upon silanization, however, predominantly large particles that are sparsely distributed on the paper fibers are observed (Fig. 2B(ii)). We can infer that the presence of fillers promotes the formation of fewer, but larger, silane-derived particles on the surface of the paper. 


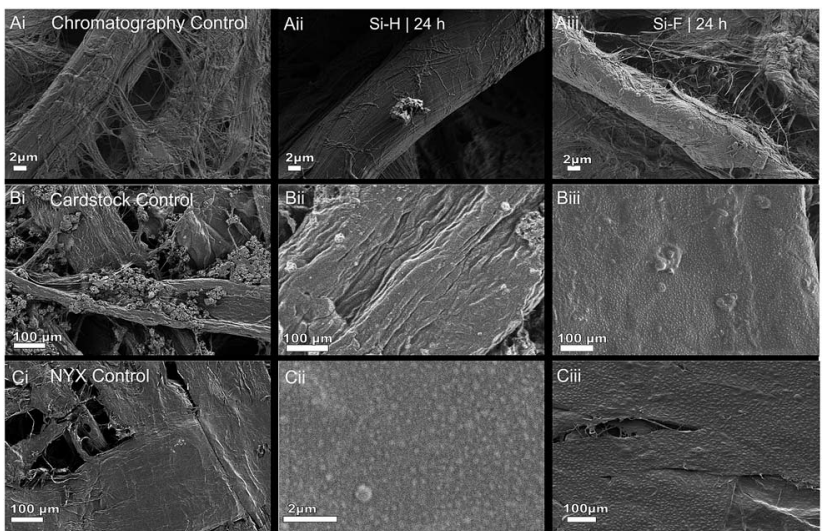

Fig. 2 Comparison of the effect of the hydrophobicity of the silane alkyl tail on surface modification and the resulting changes in texture, with respect to fillers ( $B$ and $C$ ) or paper grammage $(A<B<C)$. The left column (i) shows the unmodified papers, the middle column (ii) shows the same papers after treatment with trichloro octylsilane, and the right column (iii) shows the samples after treatment with the perfluorinated analog of the trichloro silane. All reactions were run for 24 hours. We inferred that the amount of adsorbed water on this paper, under these reaction conditions, falls outside the limits of the critical gel formation coefficient, $\alpha_{\mathrm{c}}$. To minimize the amount of adsorbed surface water, we chose two main variables: (i) presence of fillers and (ii) altering the grammage (fiber density).

\section{Effect of fiber density}

To evaluate the effect of high fiber density, NYX blotting paper-a thin, transparent \{NOTE: High fiber density renders the paper translucent due to uniformity in refractive index and decreased scattering. We used this characteristic of optical properties of paper to infer that the NYX, as in the custom made paper, had a higher fiber density. The presence of inorganic fillers, however, led to a decrease in the overall density and grammage of the paper while in cardstock paper fillers (PCC) the vice versa is true. (density $\sim 0.56$ \{NOTE: predicted density $\sim 1$ as compared to the custom made paper (density $1.07 \mathrm{~g}$ $\mathrm{cm}^{-3}$ ). We infer the difference to be due to filler materials $\mathrm{g}$ $\mathrm{m}^{-3}$ vs. $0.53 \mathrm{~g} \mathrm{~m}^{-3}$ for chromatography) paper-was treated with the silane. Fig. 2C(i) shows the structure of the native NYX blotting paper, and Fig. 2C(ii) shows the same paper upon treatment with an alkyl silane. We observed that when this paper was treated with an alkyl silane, a large number of stochastically distributed particles were formed on the paper fibers. The chromatography paper, however, does not show any changes in surface morphology (Fig. 2A(i) and A(ii)). This suggests that the grammage may have a significant effect on the evolution of surface morphology upon treatment with the silane.

\section{Effect of the hydrophobicity of the $\boldsymbol{n}$-alkylsilane hydrocarbon tail}

We hypothesized that the morphology and evolution of the surface texture will be highly dependent on the hydrophobicity of the alkyl fragment of the silane used. When a lower-surfacetension perfluoroalkyl silane (interface comparable to that of
Teflon, surface energy $\left.\sim 18.5 \mathrm{mN} \mathrm{m}^{-1}\right)^{53}$ is used to functionalize the paper (surface tension $\sim 72 \mathrm{mN} \mathrm{m}^{-1}$ ), ${ }^{54}$ it is expected that the resulting polymer particle will vary in size and shape as a result of accompanying self-assembly. ${ }^{26,32,55,56}$ As the reagents polymerize, the fluorinated tails are expected to readily cluster due to the high interfacial surface energy between them and the bound water. ${ }^{49,57}$

Exposure of the Whatman chromatography \#1 paper to either the n-octyl- or perfluoro octylsilane showed no significant changes in surface texture (Fig. 2A(ii) and A(iii)). Treatment of cardstock paper with either reagent shows a loss of the filler material and, for perfluoro silane, a combination of large and small particles (Fig. 2B(ii) and B(iii)). Similarly, treatment of NYX blotting paper shows changes in surface texture for both reagents, albeit with differences in quantities between the $n$-octyl- and perfluoro silane, with the former showing a significantly higher number of particles per unit area, whereas the latter shows a confluence of the particles (Fig. 2C(ii) and C(iii)). We can therefore infer that, immediately after the reaction initiates, the perfluorinated reagent leads to small particles that rapidly grow to quickly attain complete coverage compared to the nonfluorinated analog.

\section{Effect of reaction temperature}

To investigate the effect of temperature on the treatment, we compared NYX blotting paper treated at room temperature and at $95{ }^{\circ} \mathrm{C}$. Fig. 3 shows the evolution of surface texture over time when the paper was modified with a perfluorinated reagent at ambient temperature (left column) and elevated temperature (right column). Comparison between the controls (Fig. 3A(i) and $\mathrm{A}(\mathrm{ii})$ ) and $5 \mathrm{~min}$ treated sample shows the formation of small particles at $95^{\circ} \mathrm{C}$ (Fig. 3E) but not at room temperature (Fig. 3B). When the reaction time is extended to $1 \mathrm{~h}$, particles are observed for both reaction temperatures, albeit being of different average sizes (Fig. 3C and F), whereas after $24 \mathrm{~h}$ the surface is fully covered by a new material and larger ( $\mathrm{mm}$ size) particles are also observed (Fig. 3D and G), especially for the room temperature treatment.

The ability to control the percentage of coverage and/or size of the particles formed by altering the hydrophobicity of the reagent, the temperature, or the reaction time further supports our hypothesis that surface-adsorbed water is likely involved in the reaction of trichlorosilanes with the paper surface. In fact, at $24 \mathrm{~h}$ reaction time, we anticipate that all the adsorbed water will be consumed and any water on the interior of the cellulose fibers can diffuse out and participate in the continued growth of the particles (Fig. 3D and G).

\section{Effect of treatment on the paper surface and structure}

Previously, Gandini and co-workers ${ }^{22}$ argued that treatment of a cellulosic material with trichloromethyl silane led to the production of "inorganic particles," presumably due to the formation of polysilicate on the surface of the material. Recently, Breedveld and co-workers similarly observed that nano- and microparticles are formed only for methyl trichlorosilane but not with any longer chains. These observations 

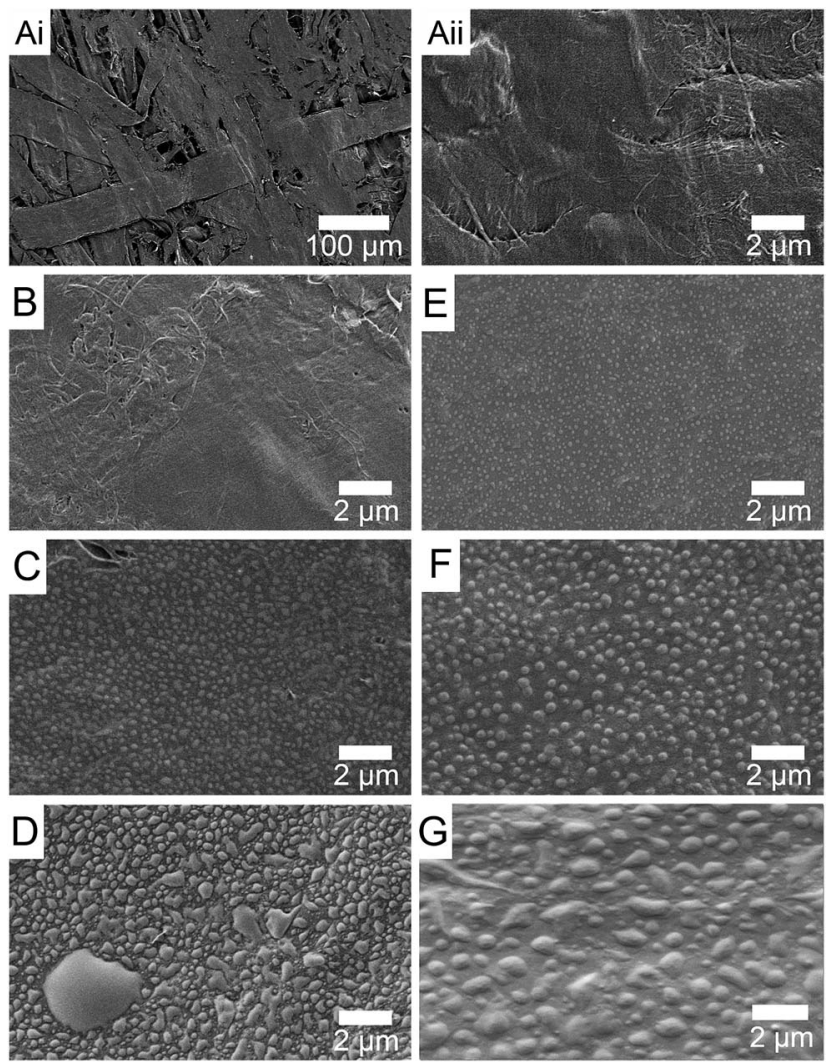

Fig. 3 SEM micrographs of surface polymerization of trichloro $(1 H, 1 H, 2 H, 2 H$-perfluorooctyl)silane on the high-grammage NYX blotting paper at room temperature (left column) and at $95^{\circ} \mathrm{C}$ (right column). (A) Control, (i) low-magnification and (ii) high-magnification images of the native paper before silanization. (B-D) show the time-course transformation of the surface texture when the polymerization is done at room temperature for $5 \mathrm{~min}, 30 \mathrm{~min}$, and $24 \mathrm{~h}$, respectively. $(E-G)$ show a time-course surface texture morphogenesis when the reaction is performed at $95{ }^{\circ} \mathrm{C}$ for $5 \mathrm{~min}, 30 \mathrm{~min}$, and $24 \mathrm{~h}$, respectively.

are not in line with a surface polymerization with concomitant self-assembly; as such, there is a need to confirm that (i) there are no significant effects of the treatment on the paper and the modification is a surface reaction, (ii) that the observed particles are organic and not due to the breakdown of the silane, and (iii) that the formed particles are actually an assembly of the polymerized silane.

(i) Bulk paper structure and organization. First, we investigated any major structural changes in the properties of the cellulose making up the paper upon chemical modification, with the inference that chemical reactions can affect the semicrystalline nature of the cellulose. To evaluate whether the alkylsilanes were diffusing into the fiber and reacting with crystalline regions of the cellulose strands, a decrease in crystallinity would be observed through wide-angle X-ray diffraction. Analysis of untreated NYX blotting paper gave two peaks at $2 \theta=15^{\circ}$ and $20^{\circ}$, suggesting that the cellulose is likely semicrystalline (see ESI, Fig. S3†). There were no observable changes in these peaks or the overall diffraction pattern, even after extended reaction times, suggesting that the reaction either was happening predominantly on the surface or did not induce a significant structural change, the former being more plausible because local structural changes will induce reorganization, and thereby decrease crystallinity.

(ii) Physical structure of the formed particles. To understand the physical form of these particles, we coated the surface of the treated (perfluoro alkylsilane, $24 \mathrm{~h}$ ) blotting paper with $10 \mathrm{~nm}$ of $\mathrm{Au}$ (to avoid surface charging) and used accelerated Ga ions in an SEM-FIB to mill through a sample of the large particles formed on the surface (Fig. 4). Fig. 4A and B are false-colorized images of two particles before and after milling (see ESI, Fig. S2, $\uparrow$ for noncolorized images). Fig. 4A(ii) shows a particle after milling away a significant section, and Fig. 4B(ii) shows two adjacent particles after milling through their midlines. We observed that the particles had solid cores, although sometimes a gap was observed near the particle-fiber interface (Fig. 4A(ii) inset), which would indicate (i) the presence of trapped gases/ gaseous by-products arising from the reaction or (ii) an asymmetry in the attachment as previously proposed by Spencer and co-workers based on spectroscopic evidence. ${ }^{37}$ We can therefore

i)
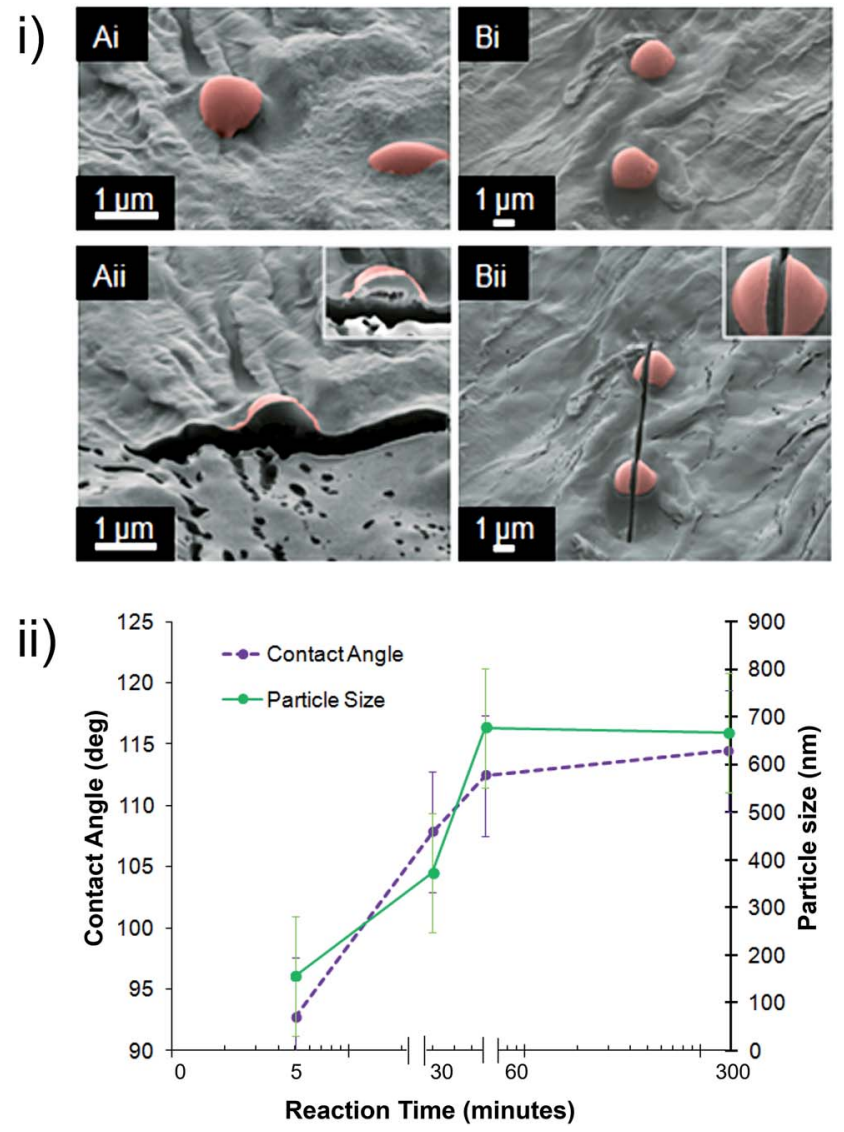

Fig. 4 SEM micrographs of large fluoroalkyl polymers before and after milling using a focused ion beam. (i) (A(i)) Two microparticles highlighted in red. (A(ii)) The largest particle was milled in half to reveal a solid core with a small gap forming underneath it (inset, $2.1 \mu \mathrm{m}$ length). (B(i)) Two particles are partially milled down the center (B(ii)) to show that the particles are solid. Inset is $2.4 \mu \mathrm{m}$ across. (ii) Correlation of particle size with hydrophobicity (static contact angle, $\theta_{\mathrm{s}}$ ) shows a gradual increase but plateaus after a $1 \mathrm{~h}$ treatment. 
infer that the particles are solid and attached to the cellulose fibers.

\section{Correlating particle size and hydrophobicity}

When the high-grammage paper NYX blotting paper was treated with fluoroalkylsilane over different reaction times (5-300 $\mathrm{min}$ ) and temperatures $\left(20^{\circ} \mathrm{C}, 70{ }^{\circ} \mathrm{C}\right.$, and $\left.95{ }^{\circ} \mathrm{C}\right)$, different sizes of particles were observed distributed on the surface of the paper, ranging from ca. $70 \mathrm{~nm}\left(5 \mathrm{~min}, 95{ }^{\circ} \mathrm{C}\right)$ to eventual particle confluence $\left(24 \mathrm{~h}, 95^{\circ} \mathrm{C}\right)$. Fig. 3 shows the growth of particles over time at a constant temperature $\left(95^{\circ} \mathrm{C}\right)$. We observed that, as predicted by step-growth polymerization kinetics, the particles grow larger with increased reaction times until they reach a point where the size seems to start decreasing as a result of confluence-that is, a film forms on the paper surface because of the merging of the growing particles. Based on the evolution in surface texture, we expect that the hydrophobicity of this material will also evolve with different reaction times.

Fig. 4(ii) shows the evolution of the average particle size and its correlation with hydrophobicity, as captured by the contact angles in NYX blotting paper. We observed that the wetting properties evolve in line with the size of the particles but plateaus after $5 \mathrm{~h}$, at which time we observed almost complete coverage of the surface with the polymeric particles. The general trend and correlation of surface changes to wetting further support the transition from "micelle-like" polymer particles to the formation of a polymer film/layer-probably what has sometimes been misinterpreted as a monolayer. The formation of the particles adds another layer of roughness to cellulose, leading to an increase in hydrophobicity; ${ }^{58}$ when the reaction time is increased, however, the number of particles per unit area decreases as a result of convergence, and surface roughness therefore decreases.

Unlike with Breedveld's and Gandini's research, we observed that with high-grammage paper, we did not attain ultrahydrophobicity. We hypothesized that failure to attain $\theta_{\mathrm{s}}>120^{\circ}$ was in part due to the inherent roughness of the paper. When lower-grammage paper was treated with the perfluorooctyltrichlorosilane, $\theta_{\mathrm{s}}=123^{\circ}$ and $\theta_{\mathrm{s}}=149^{\circ}$ were observed for Whatman chromatography \#1 and filter paper \#1 (see ESI, Fig. S8 $\dagger$ ), respectively, even though no particles were formed. The evolution of wetting properties is therefore dependent on the native roughness of the paper, and texture evolution due to the growing particles creating multiple levels of roughness, a key parameter in wetting behavior. ${ }^{58}$

\section{Chemical characterization of the particles}

Comparison of the ATR-FTIR (attenuated total reflectance Fourier transform infrared) spectra of the treated and untreated samples of the NYX blotting paper showed a slight decrease in the $-\mathrm{OH}_{\text {str }}$ peak $\left(3330 \mathrm{~cm}^{-1}\right){ }^{59}$ with a concomitant slight increase in the $\mathrm{C}-\mathrm{H}_{\text {str }}$ peak $\left(2950 \mathrm{~cm}^{-1}\right)$, upon treatment for $1 \mathrm{~h}$ (see ESI, Fig. S4 $\dagger$ ). A significant amount of the $-\mathrm{OH}_{\text {str }}$ peak was, however, still present in the treated sample, suggesting that the hydrogen bonding network in the bulk of the paper was still intact. The slight decline in the intensity of the $-\mathrm{OH}_{\text {str }}$ peak (see the inset in Fig. S4 in the ESI $\dagger$ ) suggests that only a small proportion of the $-\mathrm{OH}$ moieties in paper is involved in the transformation, which further supports the hypothesis that the reaction is likely a surface phenomenon.

Analyzing the surface by X-ray photoelectron spectroscopy (XPS) gave us the nature of the chemical species on the paper before and after treatment (Fig. 5). We have assigned our best-fit estimates of the species associated with each XPS peak and supported our assignments with literature values. ${ }^{60-62}$ Two carbon peaks are expected for cellulose corresponding to $\mathrm{C}-\mathrm{O}$ and $\mathrm{O}-\mathrm{C}-\mathrm{O}$, which we assigned to $287.9 \mathrm{eV}$ and $289.4 \mathrm{eV}$, respectively (Fig. 6A). Additional carbon signals found in the $\mathrm{C}-\mathrm{O}-\mathrm{C} / \mathrm{C}-\mathrm{OH}$ and lower binding energy peaks have been attributed to lignin and adventitious species. The ratio of oxygen to carbon helps us determine the amount of lignin in the paper. Pure cellulose will give an $\mathrm{O} / \mathrm{C}$ ratio of 0.83 , whereas machine-milled lignin gives an $\mathrm{O} / \mathrm{C}$ ratio of $\sim 0.33 .^{62}$ The
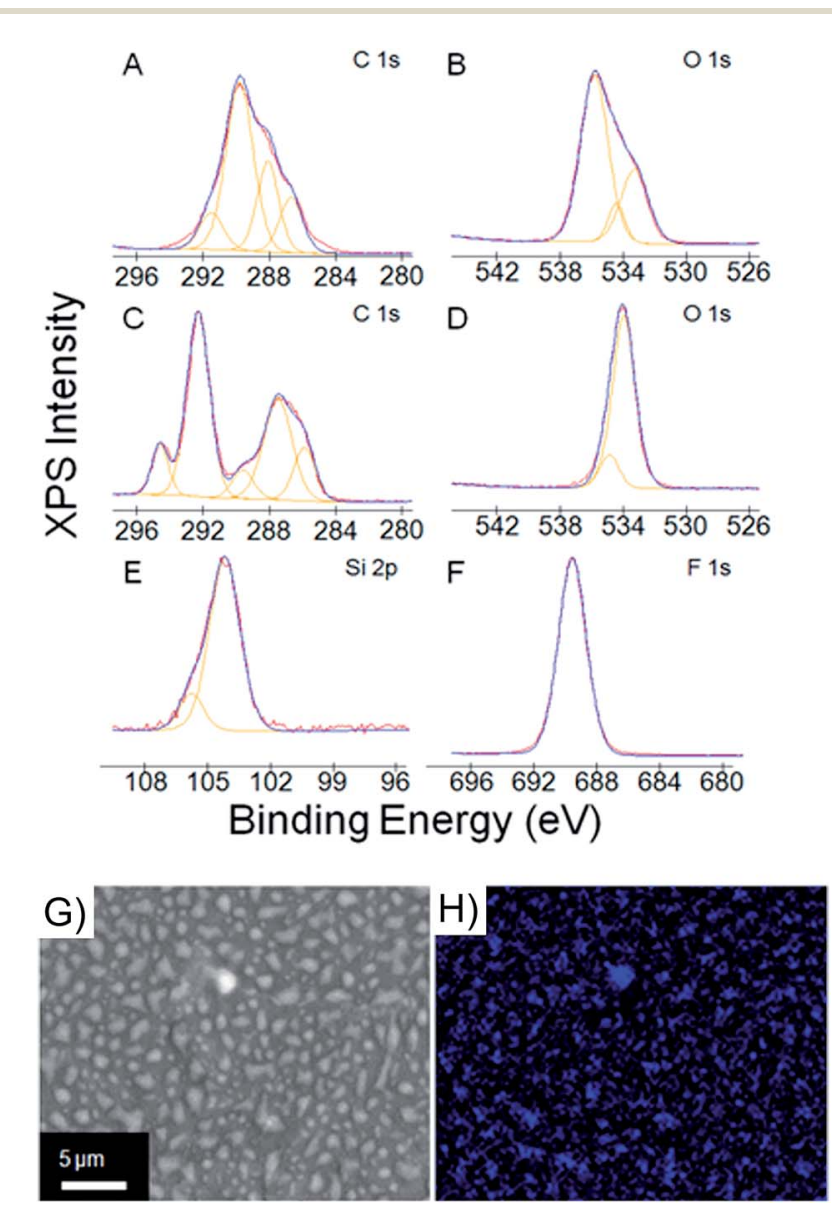

Fig. 5 (A-F) Chemical characterization of the modified paper by (G) XPS and SEM and (H) EDS. (A) Carbon and (F) oxygen signals from the untreated paper; no fluorine was observed, and only trace amounts of adventitious Si $(<1 \%)$ were observed. (C) Carbon and (D) oxygen peaks are significantly changed upon treatment, with new peaks appearing at higher binding energies, and the peak at $290 \mathrm{eV}$ significantly decreases. The peaks that correspond to the binding energies of $(E)$ silicon and (F) fluorine are observed only on the treated sample. Correlation of the spatial position of particles with that of fluorine based on SEM and EDS data confirms that these particles are derived from the silane. 
A
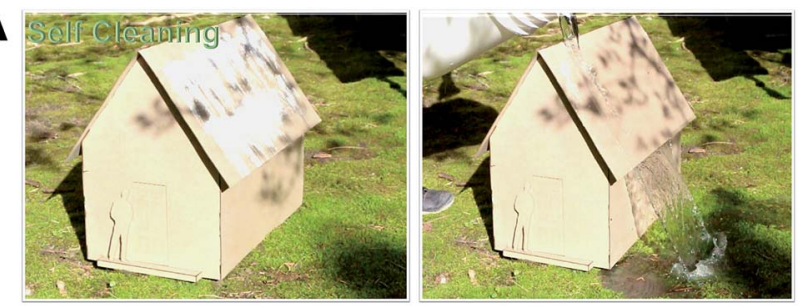

B

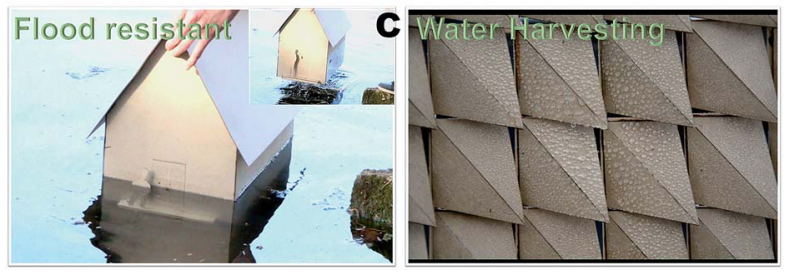

Fig. 6 Potential applications of treated hydrophobic paper. (A) Semipermanent structures: model cardboard houses are protected from water, and any particulate washes away on contact with flowing water. (B) Soaking the model house in water does not compromise hydrophobicity. (C) Water harvesting or flow control: with appropriate design, paper-based structures can be used for water harvesting or controlling the flow of a condensate.

untreated blotting paper sample comes at a ratio of 0.75 , which corresponds to a milled wood lignin concentration of $\sim 10 \%$. In addition to the presence of lignin, the presence of adventitious carbon challenges any firm inferences that can be drawn from the $\mathrm{C} / \mathrm{O}$ ratio, and as such we exercise caution in interpreting these peaks. In the O1s region of the XPS, there are two discernible peaks at $533.8 \mathrm{eV}$ and $531.7 \mathrm{eV}$, which we have assigned to $\mathrm{C}-\mathrm{OH}$ and $\mathrm{C}-\mathrm{O}-\mathrm{C}$, respectively (Fig. 5B). From the XPS data, we observed that prior to treatment, the paper had adventitious silicon (0.68 atomic\%). Upon treatment with trichloro fluoroalkyl silane, there were significant changes in the C1s and O1s regions. The new C1s peaks at $290.9 \mathrm{eV}$ and 293.2 $\mathrm{eV}$ were assigned to $\mathrm{CF}_{2}$ and $\mathrm{CF}_{3}$ with an atomic ratio of $\sim 5: 1$, which matches the expected ratio for the trichloro fluoroalkyl silane (Fig. 5C). The two remaining signals from the nonfluorinated methylene groups overlap with cellulose signals and cannot be isolated through peak deconvolution. The $289.8 \mathrm{eV}$ peak decreases upon treatment because surface $\mathrm{C}-\mathrm{OH}$ groups react with the alkyl silane. The original O1s peaks both shift and converge to a single peak at $532.8 \mathrm{eV}$ (Fig. 5D). The oxygen in polydimethylsiloxane is in an electronic environment almost analogous to that of the polymerized fluoroalkylsilane and is expected to appear at $532.0 \mathrm{eV},{ }^{60}$ but with the addition of a fluorinated alkyl group, the peak shifts to $532.8 \mathrm{eV}$. The peak at $688.2 \mathrm{eV}$ (Fig. 5F) matches the literature values for organic fluorine and has an atomic ratio to silicon of $13: 1$. This is the expected $\mathrm{F} / \mathrm{Si}$ ratio, suggesting that there was no X-ray beam damage, a known issue with fluoropolymers. The silicon peaks come at $103.0 \mathrm{eV}$, which we assign to organic silicon bound to oxygen (Fig. 5E). No peaks were found for chlorine, so this silicon peak is assigned to organic Si-O. The XPS data are consistent with fluorinated alkyl silane adhering to a cellulose surface.

The XPS supports the presence of the polymerized fluoroalkyl silane on the paper but does not directly assign the elemental composition to the particles. Clearly, there are particles growing on the surface of the paper, but the source of the particles has not been definitely identified. Energy-dispersive X-ray spectroscopy (EDS) was used to map the locus of key elements in treated (trichloro fluoroalkyl silane) and untreated blotting paper. Because of the high energy needed for EDS elemental mapping, a thin layer of gold was needed to prevent charging and minimize surface damage. Fig. $5 \mathrm{H}$ gives the elemental maps of fluorine in the treated paper (see ESI, Fig. $\mathrm{S} 7, \dagger$ for $\mathrm{C}, \mathrm{O}$, and $\mathrm{Si}$ ), whereas for the untreated paper no $\mathrm{F}$ was observed (see ESI, Fig. S6†). For the treated paper, as expected, $\mathrm{C}$ and $\mathrm{O}$ are ubiquitously distributed (see ESI, Fig. $\mathrm{S} 7 \dagger$ ), while $\mathrm{F}$ is aligning with particle distribution-that is, there is a strong overlap between the location of $\mathrm{F}$ and the particles. The slight decline in the intensity of the F signal can be attributed to damage from the strong X-ray beam needed for this analysis. In the control paper sample, adventitious silicon is observed, and thus the lack of a strong correlation between $\mathrm{Si}$ maps and particle distribution. The elemental maps therefore support the formation of fluoroalkyl silane polymer particles with regions of untreated paper between them. We can therefore infer that the paper is probably biphobic - that is, there are regions that are hydrophobic (fluorinated) and hydrophilic (nonfluorinated).

\section{Potential applications-self-cleaning structures}

Paper is rapidly emerging as a renewable, sustainable, green structural material. ${ }^{63}$ The classic work of Shigeru Ban illustrated that paper can be used to build emergency temporary houses/ structures. ${ }^{63}$ Drawing inspiration from Ban's work, we demonstrate, using model structures, that incorporation of chemical treatment allows these structures to be resilient and, as such, extends their lifetimes. Fig. 6 shows the resistance of model structures to water. In Fig. 6A, a model structure of a house is smeared with white clay powder that is then readily cleaned off by pouring water over the structure. We also demonstrate that the paper structures can withstand water damage such as that sustained through flooding (Fig. 6B). These results indicate that the treated paper is self-cleaning.

Having created biphobic material akin to the Namib beetle, albeit of opposite configuration (hydrophobic bumps on the hydrophilic surface), we hypothesized and demonstrated (Fig. 6C) that the hydrophilic trenches can condense water until such a point that the droplet grows and rolls off due to capillary forces and contact with the hydrophobic bumps. This process is analogous to that utilized by the Namib beetle, ${ }^{1-3}$ except that in lieu of gravity, we exploited the felicitous design and capillary effects to facilitate droplet coalescence and roll-off. In addition to traditional structures, we demonstrated that the treatment can be used to prepare functional smart-surfaces structures, as demonstrated in Fig. 6C, which is used for water harvesting.

In addition to the use of the silane treatment to advance and diversify the applications of lignocellulosic materials shown here, we recently demonstrated that this treatment delays biodegradation, allowing paper to remain buried under ambient soil conditions for about a year before being fully biodegraded, ${ }^{64}$ and upon incineration, the paper does not emit 
$\mathrm{HF}^{66}$ a potential deleterious by-product of using silanes. Combining the degradation studies with the current work, we believe that a felicitous choice of treatment conditions will diversify the applications of paper and other lignocellulosic materials.

\section{Conclusions}

Functionalization of surfaces to impart hydrophobicity can be complicated by the presence of adventitious components and/ or surface-adsorbed water. Considering the presence of adsorbed water, we demonstrated or inferred the following.

\section{Mechanism of the reaction of trivalent alkylsilanes with polar surfaces}

Following the nature of the adsorbed layer, we can summarize the process of attachment of the silane as follows: (i) loosely bound water and ambient water vapor are removed on application of vacuum, although at $\sim 30 \mathrm{mmHg}$, there is still a significant amount of water left on the surface. (ii) Following Samyn's ${ }^{57}$ definition of the different types of water, we expect that the initial reaction of the silane with the surface will lead to cross-linking and deposition of growing oligomeric silanes on the surface. Because the bound water layer is mobile, the oligomerizing silanes can move around to form larger aggregates. (iii) Upon further depletion, the static layers of bound water (socalled freezing or icelike layers) are exposed and are less mobile than the capillary water. Local depletion is therefore possible, leading to local attachment of the silane macromonomer on a surface hydroxyl group. (iv) The polymerized silane particle continues to grow as more silanes are attached to its edges. (v) As the particle coverage tends to confluence, integration of small particles will lead to larger ones and eventually to full confluence. The surface is then covered with a film, not a monolayer, of the silane. The resulting surface acquires a rough (rugged) texture because of the spherical nature of the growing particles-monolayers would lead to a texture that is dictated by the substrate. ${ }^{65}$

\section{Fabrication of a biphobic surface with hydrophobic bumps and hydrophilic trenches}

We demonstrate the fabrication of these types of surfaces by grafting polymeric particles on the surface of a paper. By mapping the distribution of hydrophobic molecular species (as captured by elemental fluorine) on the surface of an otherwise hydrophilic surface, we infer that the surface will have high and low surface energy regions. Because these types of surfaces have hydrophobic bumps and hydrophilic "trenches," we refer to them as i-NB (inverse Namib beetle) surfaces, as they are an inversion of those observed on the Namib beetle.

Surface-bound water can be used as a co-monomer in gel formation step-growth polymerization of trichloro silanes

We hypothesized and demonstrated that because most surfaces have surface-bound water, by employing reagents that react with water, one can generate a polymeric material recruiting bound water as a comonomer. Because the quantity of adsorbed water depends on the surface energy and/or architecture of the material, tuning the fiber density and distribution can lead to different amounts of water and thus to differences in particle distribution. We demonstrated that by paying attention to the grammage and fillers in a type of paper, the product of a simple silanization can give two very different results. We trace this difference to the amount of adsorbed water and the role of the fillers in the ensuing surface polymerization.

\section{Surface texture can be engineered through reaction kinetics}

From the Carothers paradox, we deduced and demonstrated that the size of particles formed on the surface of the paper can be tuned. Through the felicitous choice of reagents, reaction conditions, and the material to modify, we demonstrated that the surface texture evolves over a large range of reaction times (24 h); hence, by exploiting the slow nature of the growth of the particles, the surface texture can be tuned. We also demonstrated that the resultant texture depends on fiber density, with too high or too low grammage leading to disordered deposition. This grammage dependence can be attributed to the correlation of exposed surface area to accessible bound water, with too low or too high fiber density, leading to a significant decrease in the amount of bound water available for surface polymerization. This inference is in line with the dependence of the gel formation coefficient, $\alpha_{\mathrm{c}}$, on the ratio of the two reacting polyvalent monomers.

\section{Wetting properties (hydrophobicity) of the paper can be tuned}

Having demonstrated that the surface texture can be tuned, by understanding that wetting properties correlate with the surface texture, we demonstrated that the hydrophobicity of the treated paper changes with particle size.

\section{Acknowledgements}

This work was supported by the Iowa State University through start-up funds. MT acknowledges support from a 'Black and Veatch building a world of difference' faculty fellowship, SO-R was partially supported by a GMAP fellowship from Iowa State University, JC was supported in part by a Catron fellowship from the Catron Solar Energy Center and BC was supported in part by the U.S. department of Energy through Ames Lab. We thank Leslie Forehand, Shelby Doyle and their studio design class for help in fabricating the paper structures. This work was carried out in part at the Center for Nanoscale Systems (CNS), a member of the National Nanotechnology Infrastructure Network (NNIN), which is supported by the National Science Foundation under NSF award no. ECS-0335765. CNS is part of Harvard University.

\section{References}

1 J. Guadarrama-Cetina, A. Mongruel, M. G. Medici, E. Baquero, A. R. Parker, I. Milimouk-Melnytchuk, W. Gonzalez-Vinas and D. Beysens, Eur. Phys. J. E: Soft Matter Biol. Phys., 2014, 37, 1-6. 
2 Y. Hou, M. Yu, X. Chen, Z. Wang and S. Yao, ACS Nano, 2015, 9, 71-81.

3 L. Zhai, M. C. Berg, F. C. Cebeci, Y. Kim, J. M. Milwid, M. F. Rubner and R. E. Cohen, Nano Lett., 2006, 6, 12131217.

4 Y. Choi, T. Brugarolas, S.-M. Kang, B. J. Park, B.-S. Kim, C.-S. Lee and D. Lee, ACS Appl. Mater. Interfaces, 2014, 6, 7009-7013.

5 T. Norgaard, M. Ebner and M. Dacke, PLoS One, 2012, 7, e34603.

6 J. L. Plawsky, J. K. Kim and E. F. Schubert, Mater. Today, 2009, 12, 36-45.

7 M. A. Shirgholami, M. S. Khalil-Abad, R. Khajavi and M. E. Yazdanshenas, J. Colloid Interface Sci., 2011, 359, 530-535.

8 E. K. Her, T.-J. Ko, K.-R. Lee, K. H. Oh and M.-W. Moon, Nanoscale, 2012, 4, 2900-2905.

9 Y. Wang, L. Zhang, J. Wu, M. N. Hedhili and P. Wang, J. Mater. Chem. A, 2015, 3, 18963-18969.

10 Y. Zheng, C. Su, J. Lu and K. P. Loh, Angew. Chem., Int. Ed., 2013, 52, 8708-8712.

11 X.-W. Li, Q.-X. Zhang, Z. Guo, J.-G. Yu, M.-K. Tang and X.-J. Huang, RSC Adv., 2015, 5, 29639-29646.

12 J.-D. Brassard, D. K. Sarkar and J. Perron, Appl. Sci., 2012, 2, 453-464.

13 W. A. Daoud, J. H. Xin and X. M. Tao, J. Am. Ceram. Soc., 2004, 87, 1782-1784.

14 B. Leng, Z. Shao, G. De With and W. Ming, Langmuir, 2009, 25, 2456-2460.

15 S. Li, S. Zhang and X. Wang, Langmuir, 2008, 24, 5585-5590.

16 M.-J. Oh, S.-Y. Lee and K.-H. Paik, J. Ind. Eng. Chem., 2011, 17, 149-153.

17 L. Zhai, F. Ç. Cebeci, R. E. Cohen and M. F. Rubner, Nano Lett., 2004, 4, 1349-1353.

18 H. Zhou, H. Wang, H. Niu, A. Gestos, X. Wang and T. Lin, Adv. Mater., 2012, 24, 2409-2412.

19 A. G. Cunha and A. Gandini, Cellulose, 2010, 17, 875-889.

20 M. L. Hair and W. Hertl, J. Phys. Chem., 1969, 73, 2372-2378.

21 E. M. James, in Silicones and Silicone-Modified Materials, ACS, 2000, vol. 729, ch. 1, pp. 1-10.

22 M. Abdelmouleh, S. Boufi, M. N. Belgacem, A. P. Duarte, A. Ben Salah and A. Gandini, Int. J. Adhes. Adhes., 2004, 24, 43-54.

23 J. G. Matisons, Silanes and other coupling agents, 2009, vol. 5, p. 3.

24 T. Textor and B. Mahltig, Appl. Surf. Sci., 2010, 256, 1668-1674.

25 Y. Xie, C. A. S. Hill, Z. Xiao, H. Militz and C. Mai, Composites, Part A, 2010, 41, 806-819.

26 C. Haensch, S. Hoeppener and U. S. Schubert, Chem. Soc. Rev., 2010, 39, 2323-2334.

27 C. Zhang, N. E. Shephard, S. M. Rhodes and Z. Chen, Langmuir, 2012, 28, 6052-6059.

28 A. C. Glavan, R. V. Martinez, A. B. Subramaniam, H. J. Yoon, R. Nunes, H. Lange, M. M. Thuo and G. M. Whitesides, Adv. Funct. Mater., 2014, 24, 60-70.

29 A. Hozumi, K. Ushiyama, H. Sugimura and O. Takai, Langmuir, 1999, 15, 7600-7604.
30 D. Devaprakasam, S. Sampath and S. K. Biswas, Langmuir, 2004, 20, 1329-1334.

31 E. Besson, A.-M. Gue, J. Sudor, H. Korri-Youssoufi, N. Jaffrezic and J. Tardy, Langmuir, 2006, 22, 8346-8352.

32 K. Wen, R. Maoz, H. Cohen, J. Sagiv, A. Gibaud, A. Desert and B. M. Ocko, ACS Nano, 2008, 2, 579-599.

33 N. Herzer, S. Hoeppener and U. S. Schubert, Chem. Commun., 2010, 46, 5634-5652.

34 V. V. Naik, R. Stadler and N. D. Spencer, Langmuir, 2014, 30, 14824-14831.

35 Z. Tang, H. Li, D. W. Hess and V. Breedveld, Cellulose, 2016, 23, 1401-1413.

36 A. G. Cunha, C. Freire, A. Silvestre, C. P. Neto, A. Gandini, M. N. Belgacem, D. Chaussy and D. Beneventi, J. Colloid Interface Sci., 2010, 344, 588-595.

37 V. V. Naik, M. Crobu, N. V. Venkataraman and N. D. Spencer, J. Phys. Chem. Lett., 2013, 4, 2745-2751.

38 A. W. Martinez, S. T. Phillips, G. M. Whitesides and E. Carrilho, Anal. Chem., 2009, 82, 3-10.

39 A. Nilghaz, D. H. B. Wicaksono, D. Gustiono, F. A. A. Majid, E. Supriyanto and M. R. A. Kadir, Lab Chip, 2012, 12, 209-218.

40 J. Yadav, M. Datta and V. S. Gour, BioResources, 2014, 9, 5066-5072.

41 S. Ban and K. Shodhan, Perspecta, 2003, 34, 154-159.

42 S. J. Risch, ACS Food Packaging, 2000, vol. 753, ch. 1, pp. 1-7.

43 N. Bordenave, S. Grelier and V. Coma, Biomacromolecules, 2000, 11, 88-96.

44 Y. S. Song, H. J. Park and V. Komolprasert, J. Agric. Food Chem., 2000, 48, 5856-5859.

45 H. Zhao, J. H. Kwak, Z. Conrad Zhang, H. M. Brown, B. W. Arey and J. E. Holladay, Carbohydr. Polym., 2007, 68, 235-241.

46 D. Ciolacu, F. Ciolacu and V. I. Popa, Cellul. Chem.Technol., 2011, 45, 13.

47 M. Abdelmouleh, S. Boufi, A. Ben Salah, M. N. Belgacem and A. Gandini, Langmuir, 2002, 18, 3203-3208.

48 M. C. Howland, A. R. Sapuri-Butti, S. S. Dixit, A. M. Dattelbaum, A. P. Shreve and A. N. Parikh, J. Am. Chem. Soc., 2005, 127, 6752-6765.

49 S. Oyola-Reynoso, Z. Wang, J. Chen, S. Çınar, B. Chang and M. Thuo, Coatings, 2015, 5, 1002-1018.

50 A. Pallandre, K. Glinel, A. M. Jonas and B. Nysten, Nano Lett., 2004, 4, 365-371.

51 R. Subramanian, H. Fordsmand and H. Paulapuro, BioResources, 2007, 2, 91-105.

52 J. Shen, Z. Song, X. Qian and W. Liu, BioResources, 2009, 4, 1190-1209.

53 D. H. Kaelble, J. Adhes., 1970, 2, 66-81.

54 W. M. Haynes, CRC handbook of Chemistry and Physics, CRC press, 2014.

55 C. S. Brazel and S. L. Rosen, Fundamental Principles of Polymeric Materials, Wiley, 2012.

56 M. Rebhan, A. Plagge, M. Rohwerder and M. Stratmann, Langmuir, 2002, 18, 2577-2581.

57 P. Samyn, J. Mater. Sci., 2013, 48, 6455-6498.

58 C. Frankiewicz and D. Attinger, Nanoscale, 2016, 8, 39823990. 
59 Y. Sun, Z. Zhang and C. P. Wong, J. Colloid Interface Sci., 2005, 292, 436-444.

60 C. G. Pantano and T. N. Wittberg, Surf. Interface Anal., 1990, 15, 498-501.

61 L. Fang, L. Chang, W.-j. Guo, Y. Chen and Z. Wang, Appl. Surf. Sci., 2014, 288, 682-689.

62 L.-S. Johansson, J. M. Campbell, K. Koljonen and P. Stenius, Appl. Surf. Sci., 1999, 144, 92-95.

63 S. Ban and R. Miyake, Shigeru Ban: Paper in Architecture, Rizzoli International Publications, New York, 2009.
64 S. Oyola-Reynoso, D. N. Kihereko, B. S. Chang, J. N. Mwangi, J. Halbertsma-Black, J.-F. Bloch, M. M. Thuo and M. M. Nganga, Ind. Crops Prod., DOI: 10.1016/ j.indcrop.2016.08.051.

65 Z. Wang, J. Chen, S. Oyola-Reynoso and M. M. Thuo, Langmuir, 2016, 32, 8230-8237.

66 S. Oyola-Reynoso, J. Chen, B. Chang, J.-F. Bloch and M. M. Thuo, RSC Adv., 2016, 6, 82233-82237. 